\title{
Fatigue Reliability Analysis on the Special Fork of the Transmission
}

\author{
Kuizhou Sun, Jinyu Zhou and Fuxian Zhu \\ Jiangsu University of Technology, Changzhou, P.R.China 213001, China \\ sunkuizhou@126.com
}

\begin{abstract}
Special fork is an important load-bearing component in the transmission structure, requiring relatively high abrasion resistance and impact toughness. The fork usually works under the conditions of impact and abrasion and the effect of alternating load, therefore it is prone to fatigue damage. The reliability of the fork could exert direct influence on the stability and safety of the vehicles. By highlighting the fact that the shifting special fork in the transmission of some type of light vehicle is suddenly broken and become invalid during the vehicle driving process, this article had made static strength analysis and fatigue lifetime expectation research from the perspectives of Finite Element Method and accumulated damage. The findings of the analysis will provide reference for the improvement of components structure, finding of the root cause of the accidents as well as the selection of the general design mechanism.
\end{abstract}

Keywords: Special Fork;Transmission; Fatigue Analysis

\section{Introduction}

The fork of the car is one of the key parts of the car's speed shifting system, playing an important role in shifting the speed and changing the direction. The fork could move the ring gear of synchronizer to separate and unite, thus the speed shifting is achieved. As a part of the car, the fork has a bearing on the safety of the car and person. The clear speed gear and the smooth transition have always been the objective of the transmission control facility's design and an important index for the evaluation of a good transmission. The ingenuous and flexible control facility could not only improve the comprehensive functions of the transmission but also promote the comfortable feeling of driving and riding. We should attach much importance to the fork, which is one of the most elemental components of the transmission control facility [1].

The property of the speed shifting of the transmission have a direct bearing on the technological level and the performance of the car. As an important part of the transmission, the fork should not only have enough stiffness and strength but also good reliability. According to the statistics, about $80 \%$ of the mechanical structure damage belongs to fatigue damage [2]. As far as the workmanship of the fork design structure is concerned, the workpiece with poor stiffness is easy to be out of shape when the force is exerted upon it. The invalidity of the fork starts from the fatigue fissure to the eventual breakage. The special fork is a obviously special compared with the symmetrical fork, it is a fork that has different shape due to the space structure. The Figure 1 is the fork part of the transmission of the car produced by some companies. 


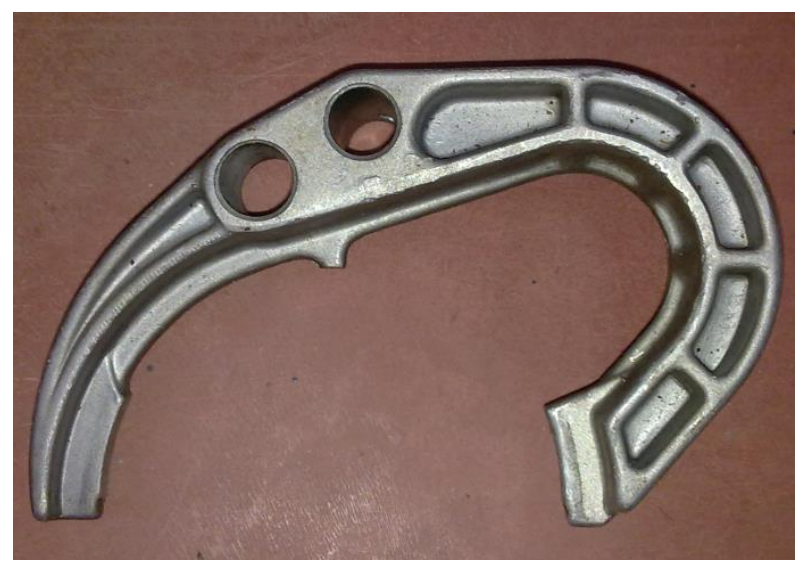

Figure 1. The Fork Part of the Transmission

During the research and development process of the car's transmission, a lot of bench tests and endurance tests would be conducted on the fork parts. It is costly and has a long period. Moreover, problems usually occur after the product design is finished, presenting some difficulties to the change of the design. Through the finite fatigue simulation analysis towards the transmission fork, the fatigue endurance forecast could be made in the early days of fork design so as to find out the weak part of the structure and come up with reasonable improvement solutions and significantly reduce or replace some fatigue tests. The fatigue simulation results could also serve as guide and reference for the design of the manufactures. With the gradual improvement of the computers' software and hardware, the design of the key part fork could turn from the fixed lifetime design to the lifetime's ration design[3].

This article adopts the nominal of fatigue analysis method and establishes finite element model and makes the static strength analysis and fatigue lifetime forecast based on ANSYS Workbench platform. It has found that the fork stress concentration and the place that fatigue damage exists are both located in the port area of the fork and the fork is verified to determine whether it meets the requirements.

\section{The Establishment of Finite Element Model}

\subsection{Modeling Software and Analysis Platform}

The UG is adopted to conduct parametric modeling. The Finite Element analysis adopts the simulation platform based on ANSYS Workbench which is the new generation CAE analysis environmental and application platform. ANSYS Workbench includes a variety of tools, namely modeling tool, analysis tool, optimization tool and gridding tool.

\subsection{Establish the Finite Element Model}

The fork is used in the manual mechanical transmission. The parametric modeling is conducted in accordance with the dimensions of the deign drawing in UG, using the port of UG and ANSYS Workbench to load the established fork three dimensional model into Workbench as shown by the Figure 2 . 


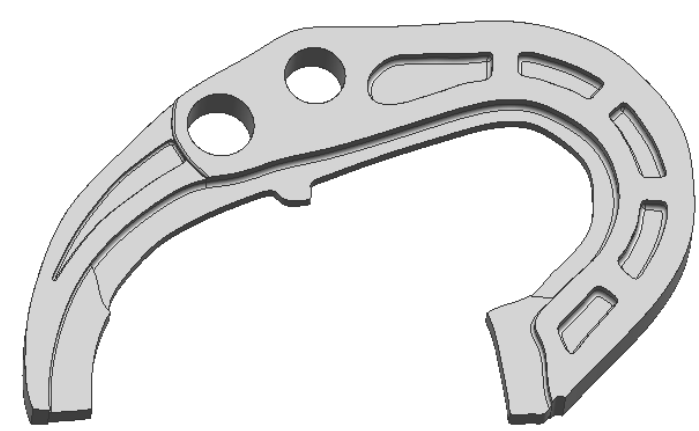

Figure 2. The Three Dimensional Model of Transmission Fork

\section{Static Force Analysis}

The static force analysis is made using the static structural module of Workbench. The material of the fork is cast steel ZG310-570, elasticity modulus E=206Gpa, Poisson ratio=0.3, Surrendering strength $=310 \mathrm{Mpa}$, Pull-resistant strength $=570 \mathrm{Mpa}$, the parts' $S-N$ curve is achieved by the calculation.

The grid is automatically divided by adopting the 10 pitch point tetrahedron element and 20 pitch points hexahedron element that are inherently determined by the Workbench. After the division of the grid, the unit is 9267 and the panel points are 17483. The fork Finite Element Model after the division of the grid is as shown in Figure 3.

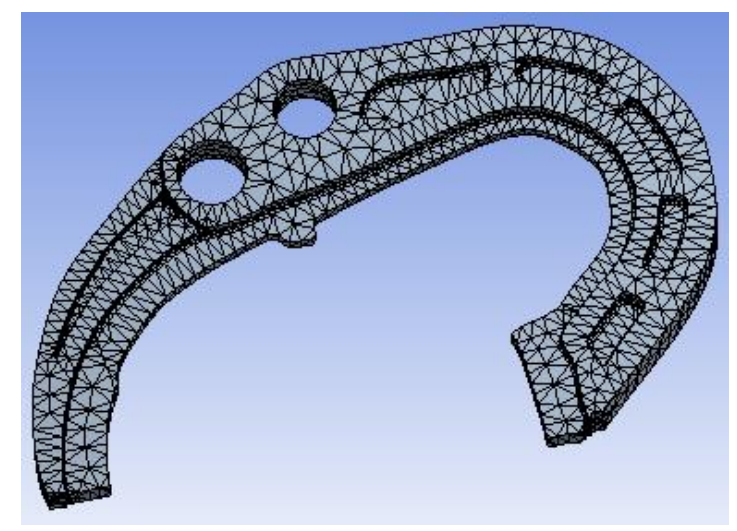

Figure 3. Fork Grid Picture

The hole fixed restraint is adopted in the Workbench due to the fact that one side of special fork is rotated around the axis and the two sides of fork claw would be repeatedly pressed with the gear end face. In accordance with the requirements of the test, the loading endured by the two sides of fork claw is alternating load, the force ranges from $420 \sim 460 \mathrm{~N}$. The maximum $460 \mathrm{~N}$ is adopted when the static force is loaded. The static force is loaded on the two sides of the fork claw, and the direction is perpendicular to the side face.

The whole shape configuration and equivalent tress cloud picture of special fork are achieved by the static force. Firstly, add total deformation and equivalent tress in the "Solution" of Workbench. Then through the calculation, the whole shape configuration and equivalent tress cloud picture of special fork are achieved. The two pictures are shown in Figure 4 and 5. 


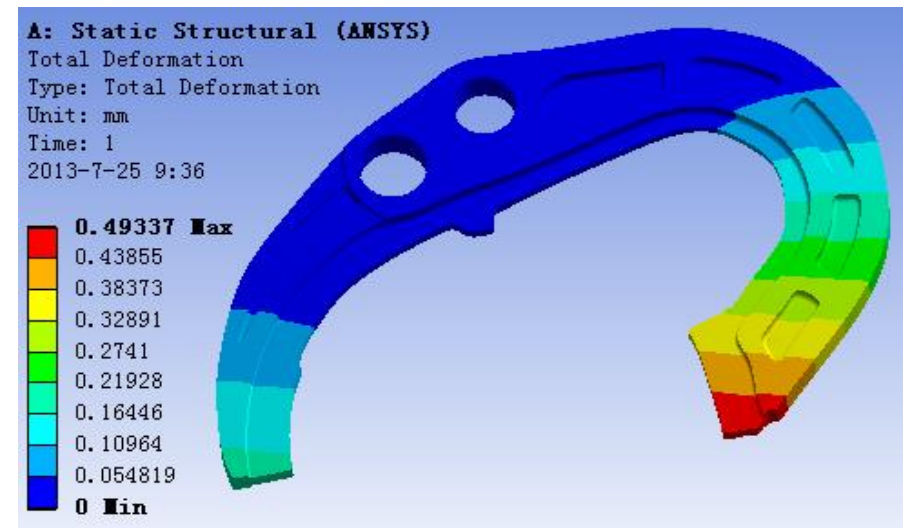

Figure 4. The Whole Shape Configuration of Special Fork

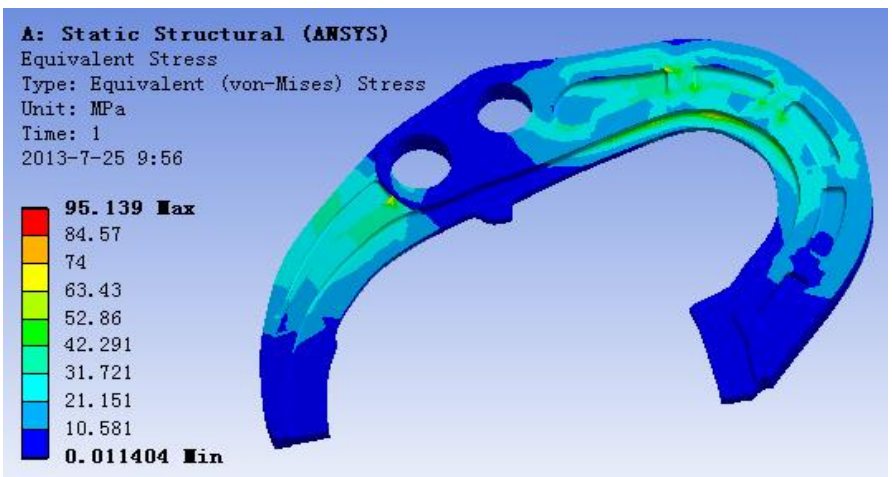

Figure 5. The Equivalent Tress Cloud Picture of Special Fork

From the static force analysis findings, it is known that the maximum shape configuration exists in the two claws, the size of which is $0.493 \mathrm{~mm}$, satisfying the design requirements. The maximum stress is 95.1 Mp, belonged in the strength scope of the cast steel ZG310-570, satisfying the design requirements, just as shown in Figure 5. The stress endured by the two sides of the two claws of the special fork is relatively bigger, the stress concentration is generated.

\section{The Factors Influencing the Extent of Fatigue}

The fatigue limit and S-N curve of the material could only represent the fatigue property of the standard and smooth sample. In fact, the size of the parts, the shape and processing surface of real fork is distinctly different from that of the standard sample. A series factors affecting the fatigue strength should be considered in determining the $S$ - $N$ curve. The factors affecting the $S-N$ curve include stress concentration, the size of the shape, the surface situation, loading type and so on[4].

The special fork material of the transmission is cast steel ZG310-570, the surrendering limit is $310 \mathrm{MPa}$, the strength limit is $570 \mathrm{Mpa}$. When the survival rate is $95 \%$, it could be found that $a=31.9285, b=-10.510$. The two numbers could be substituted in the expression of the curve:

$$
\lg N=31.9285-10.5101 \lg \sigma_{-1}
$$


In the expression, $\sigma_{-1}$ is referred to as the bending fatigue limit.

The bending fatigue strength decreasing coefficient could be calculated first.

$$
k_{\sigma D}=\frac{k_{\sigma}}{\varepsilon}+\frac{1}{\beta_{1}}-1
$$

In the expression, $k_{\sigma D}$ is the bending fatigue strength decreasing coefficient; $\beta_{1}$ is the surface machining factor; $\varepsilon$ is size factor; $k_{\sigma}$ is effective stress concentration factor.

$$
k_{\sigma}=1+q\left(\alpha_{\sigma}-1\right)
$$

In the expression, $q$ is sensitivity coefficient; $\alpha_{\sigma}$ is theoretical stress concentration factor.

From the Mechanical Design Manual, the following could be achieved:

$$
q=0.5 ; \quad \alpha_{\sigma}=1.5 ; \quad \beta_{1}=1.5 ; \quad \varepsilon=0.6718 ;
$$

From the above parameters it could be found that $k_{\sigma}=1.25$.

$$
k_{\sigma D}=\frac{k_{\sigma}}{\varepsilon}+\frac{1}{\beta_{1}}-1=\frac{1.25}{0.6718}+\frac{1}{1.5}-1=1.12
$$

when $N=1 \times 10^{3}$ is substituted in the expression, it could be found:

$$
\begin{gathered}
\sigma_{-1 N}=488.4 M P_{a} \\
\sigma_{-1 N}^{\prime}=\sigma_{-1 N} / k_{S N}=488.4 / 1.1=444 M P_{a}
\end{gathered}
$$

In the expression, $\sigma_{-1 N}$ is the Bending fatigue limit after $\mathrm{N}$ times of bending; $k_{S N}$ is the coefficient of dispersion. When $N=1 \times 10^{3}, 1.1$ is taken, when $N \geq 1 \times 10^{5}, 1.4$ is taken, and the influence of $k_{\sigma D}$ should be considered. When $N=1 \times 10^{6}$, it could be calculated that $\sigma_{-1 N}=275 \quad .7 M P_{a} ;$

$$
\sigma_{-1 N}^{\prime}=\frac{\sigma_{-1 N}}{k_{\sigma D} k_{S N}}=\frac{275.7}{1.495 \times 1.4}=131.72 M P_{a}
$$

When $N=1 \times 10^{3}$ and $N=1 \times 10^{6}$, the stress value are entered in the two logarithm coordinate system, and the straight line is connected, so the $S$ - $N$ curve is achieved, just as shown in the Figure 6. Enter the above $S-N$ curve into the material fatigue curve of the ANSYS Workbench.

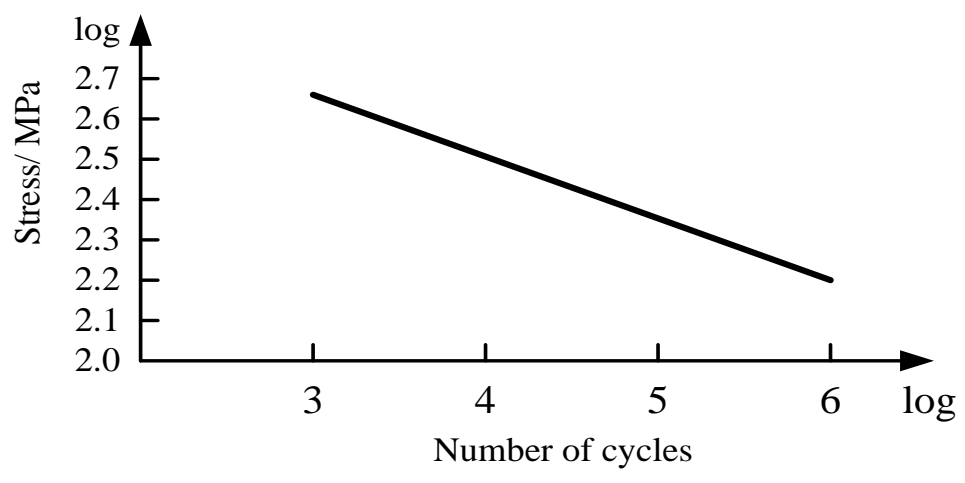




\section{Figure 6. The Simplified S-N Curve of the Parts}

The root cause for fatigue fracture is the component of stress also named mean stress, which also has some influence on the fatigue limit. Within a steady range of stress, the condensed steady stress improves the fatigue limit while the stretched static stress reduce the fatigue limit. it is generally acknowledged that residual stress has the same influence on the fatigue limit with mean stress[5]. For the same material, we can draw up its chart of fatigue limit according to its fatigue limit under the mean stress ratio or stress ratio $\mathrm{R}$.

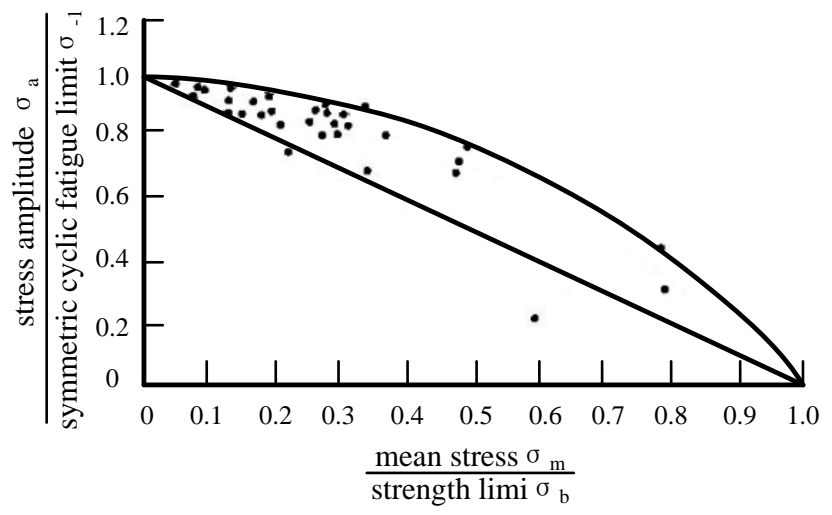

\section{Figure 7. The Fatigue Limit Graph of the Steel with the Cycle Life of 107 Times}

In Figure 7, the abscissa represents the ratio of mean stress $\sigma_{m}$ (or residual stress) and the strength limit $\sigma_{b}$, and the ordinate means the ratio of stress amplitude $\sigma_{a}$ and symmetric cyclic fatigue limit $\sigma_{-1}$, both of them being nondimensional measure. From the picture, most of the test data points fall over between the straight line and the curve, this straight line being Goodman line shown in formula (4), and the curve being Gerber parabola shown in formula (5). We get the Soderberg line with yield strength limit $\sigma_{s}$ replacing $\sigma_{b}$, which is shown in formula (6). We get Morrow line with the breaking stress $\sigma_{f}$ replacing $\sigma_{b}$, which is shown in formula (7).

$$
\begin{array}{lll}
\text { Goodman line } & \sigma_{a}=\sigma_{-1}\left(1-\frac{\sigma_{m}}{\sigma_{b}}\right) \\
\text { Gerber line } & \sigma_{a}=\sigma_{-1}\left[1-\left(\frac{\sigma_{m}}{\sigma_{b}}\right)^{2}\right] \\
\text { Soderberg line } & \sigma_{a}=\sigma_{-1}\left(1-\frac{\sigma_{m}}{\sigma_{s}}\right) \\
\text { Morrow line } & \sigma_{a}=\sigma_{-1}\left(1-\frac{\sigma_{m}}{\sigma_{f}}\right)
\end{array}
$$


In this expression, $\sigma_{a}$ is the stress amplitude of non-symmetrical cycle, $\sigma_{-1}$ is the symmetric cyclic fatigue limit, $\sigma_{m}$ is the average stress values of non-symmetrical cycle, $\sigma_{b}$ is static strength limit of the materials, $\sigma_{s}$ is the yield strength limit, $\sigma_{f}$ is the breaking stress.

Mean stress has a great influence on the fatigue lifespan of the special fork and thus needs to be corrected, and the main correction theory is Goodman, Gerber,Soderberg and Morrow theory[6]. Goodman line is a bit conservative and simple as well convenient for the ductility mental, and is used most widely in the fatigue design. Given the conservativeness of the theory computation of Goodman and the common use in the engineering practice as well as the correspondence to the mean stress correction of fatigue analysis of special fork, so Goodman formula is applied to correct the mean stress of special fork, shown in Figure 8. And also, Goodman formula is suitable for high-strength steel. Besides that, its correction result coincides with physical truth in much degree and the computation result is declined to be secure [3], so Goodman formula here is chosen to correct the mean stress.

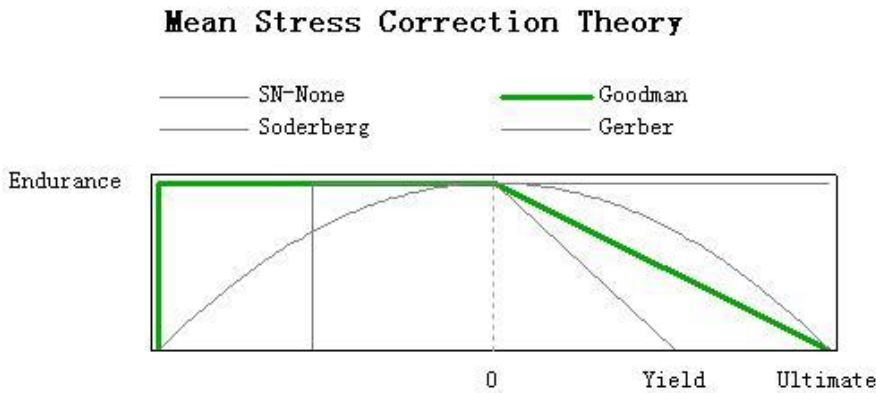

Figure 8. The Graph of Goodman Mean Stress Correction

With the maximum shifting resistance loading on the two flanks of the pusher dogs in the vertical direction, we can get the cloud picture of special fork lifespan, damage, safety factor and the graph of fatigue sensitivity, shown in Figure 9-12.

The cloud picture of special fork lifespan reveals the cycle index until the special fork get out of work due to the fatigue effect, the numerical value representing the highest cycle index the special fork can reaches under the function of loading spectrum. If the equivalent alternating stress is lower than the lowest equivalent alternating stress defined by $S-N$ curve, then we use the highest cycle index in the $S-N$ curve as the lifespan of special fork[7]. The maximum lifespan is 1E6 in the $S$ - $N$ curve of the special fork, then the default of infinite lifespan is 1E6. Just like the Figure 9 shows that the maximum lifespan of the special fork is 1E6, most areas averages 1.663E5. In that circumstance, fatigue damage won't occur. The part with lowest lifespan is in the rounding of reinforcing rib at the two flanks by the two push dogs, where fatigue damage would probably occur [8]. 


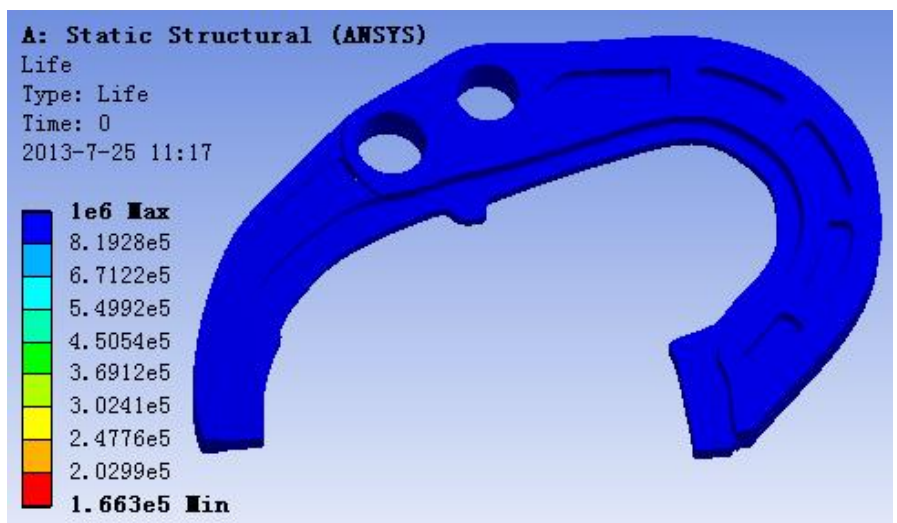

\section{Figure 9. The Cloud Picture of the Special Fork Lifespan}

The cloud picture of damage reveals ratio between the designed life of special fork and the available life. If the numerical value of damage is lower than 1 , it means that fatigue damage won't occur, and on the contrary, if the numerical value of damage is bigger than 1, then it will have been out of work before it reaches the designed life[9]. Just like Figure 10 shows the biggest numerical value of damage is 1.383 and the broken area is at the joint of the push dogs on the left and the rounding of the left hole, so the joint part is easily to get out of work while the other areas not.

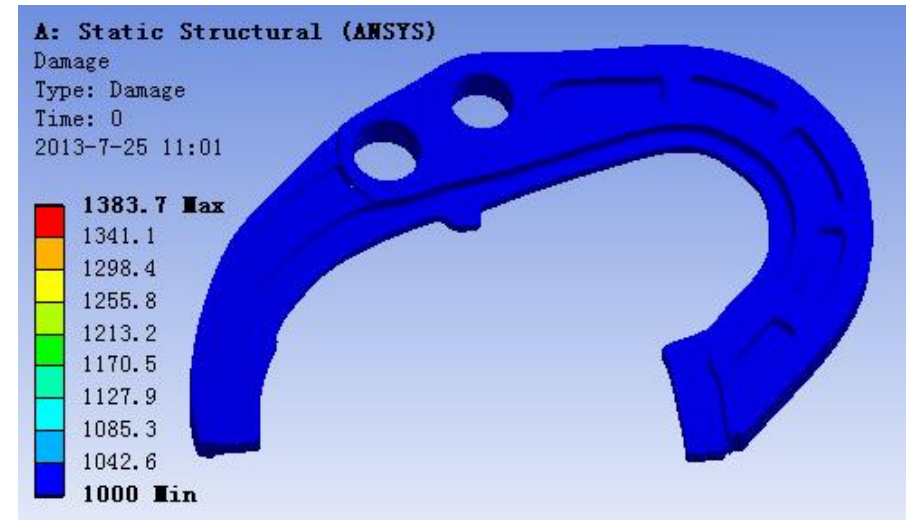

Figure 10. The Damage Picture of the Special Fork

The cloud picture of safety factor reveals the ratio between the failure stress and the designed stress of the special fork materials[10]. With the failure given the designed life and 1 $\times 10^{5}$ cycle indexes as the numerical value of designed life, we get the safety factor by calculation (the maximum is 15 ), shown in the Figure 11. From the figure, we can see the lowest numerical value of the safety factor can still reaches 1.0174 with the condition of such a high designed life. The number shows that the numerical value is high enough to satisfy the design demand and the stock utilization is comparatively high. 


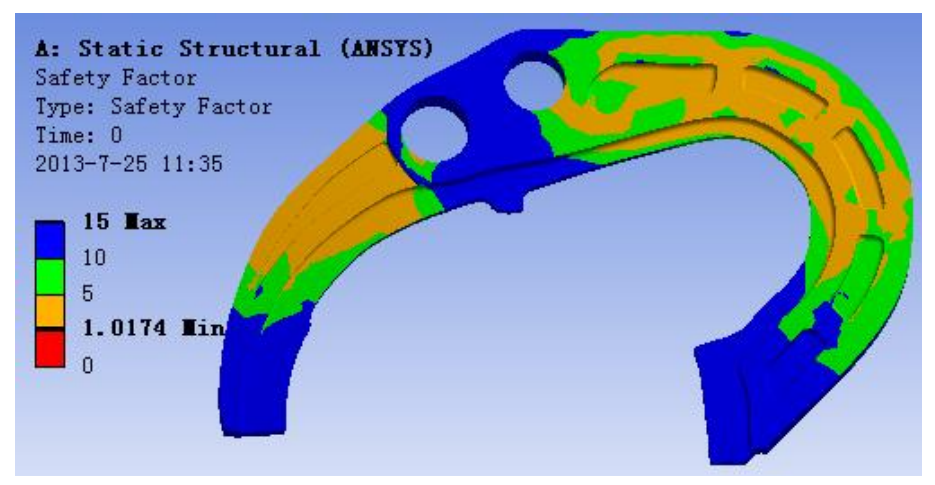

Figure 11. The Cloud Picture of Safety Factor

The curve graph of the fatigue sensitivity shows the situation of the special fork lifespan, damage or the safety factor with the load changing at the critical zone[11], shown in the Figure 12.

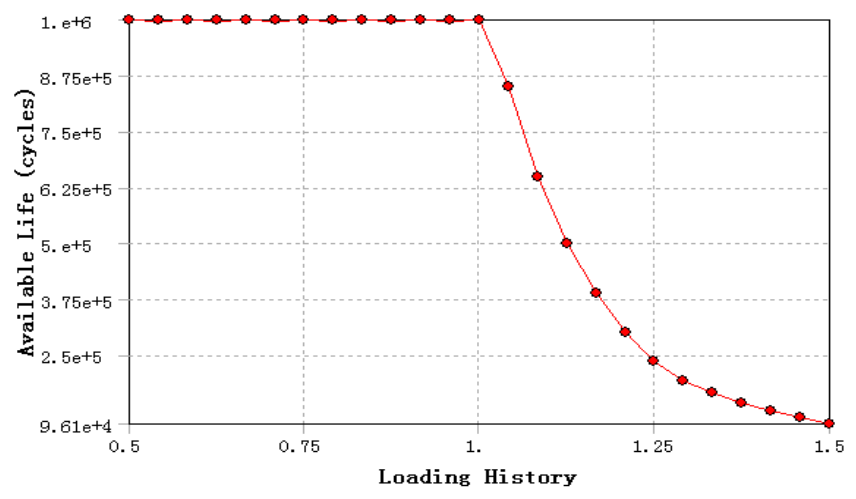

\section{Figure 12. The Curve Graph of the Fatigue Sensitivity}

\section{Conclusion}

Through the statics analysis of the special fork of automotive transmission with finite element method based on the platform-ANSYS Workbench, we get the deformation of the special fork, the numerical value of stress intensity and its location as well as the location of stress intensity concentration of the special fork - the rounding of the reinforcing rib flanked by the two pusher dogs. Meanwhile, through the fatigue lifespan analysis of the special fork, we get the numerical value of its lifespan, damage, safety factor as well as the curve of fatigue sensitivity. And also we can get the numerical value of the lifespan of the special fork and its location of damage - the rounding of the reinforcing rib at the two flanks by the two pusher dogs, thus we can draw a conclusion that the rounding of the reinforcing rib at the two flanks by the two pusher dogs are the places where both of the special fork stress concentration and fatigue damage occur.

The simulation of the fatigue lifespan of the special fork and its damage prove that its designed fatigue lifespan meets the demand and provides the basis and reference for the manufacturers to further improve the design and plays an important role in the follow-up improved design. The stimulation of the fatigue of the special fork being accordant with the actual damage speaks well that the simulating calculation of the special fork can 
comparatively correctly predict the fatigue damage part and thus is favorable to improve the design efficiency and lower R\&D costs as well as shortening the R\&D cycle.

\section{Acknowledgements}

Fund program: Basic generic technology of green manufacturing.Part project: Study of model product security of operation and life prediction technique (National High Technology Research and Development Program of China (863 project)) . No. 2012AA040104.

\section{References}

[1] W. Cian, W. Ting, "Based on ANSYS Workbench for ZL50 Wheel Loader Front Frame Fatigue Life Analysis", Beijing Automotive, vol. 6, (2011), pp.40-43.

[2] Z. Zhengtao, D. Chenghui, W. Lang, Z. Yuhua, "FEM Analysis on the Drive Axle Housing of a Jiangling Vehicle”, Automotive Engineering, vol. 29, no. 10, (2007) ,pp.896-899.

[3] Q. Dianwei, Y. Jianqiang, "Design of Combining Sliding Mode Controller for Overhead Crane Systems", International Journal of Control and Automation, vol. 6, no. 1, (2013), pp.131-140.

[4] Atul Kumar, Dheeraj Kumar, U. K. Singh, P. S. Gupta and Gauri Shankar, "Optimizing Fibre Optics for Coal Mine Automation", International Journal of Control and Automation, vol. 4, no. 3, (2011), pp.19-30.

[5] W. Weizhen, D. Yuping, Z. Shuxia, S. Hongyu, D. Wei, "The Fatigue Analysis about the Flat - die Based on ANSYS Workbench", Research on Agricultural Mechanization, vol. 9,(2011), pp.81-84.

[6] L. Xianfeng, Y. Jianwei, J. Zhixuan, "Fatigue Analysis for Spring Plate of Vehicle Shock Absorber Based on Workbench", Journal of Beijing University of Civil Engineering and Architecture, vol. 28, no. 2, (2012), pp.50-55.

[7] S. Penfei, T. Linyang, W. Yu, M. Haigang, S. Changjuan, "Fatigue Analysis of Hydraulic Support Based on Ansys oftware", Coal Mine Machinery, vol. 32, no. 11, (2011), pp.102-103.

[8] C. Demin. L. Xueyuan, H. Jibin, Y. Shihua, "Fracture of Shifting-yoke in the Gear-box for a Wheeled Chariot Based on Miner's Accumulated Damage Theory", Transactions of Beijing Institute of Technology, vol. 26, no. 10, (2006), pp.855-858.

[9] W. Ruiming, Z. Xiaojun, Z. Mingyan, P. Mingqing, "Fatigue Testing and Analysis of Automotive Driving Axle”, Automotive Engineering, vol. 25, no. 3, (2003), pp.283-286.

[10] C. Shusen, D. Bin, M. Sujun, L. Xiaohong, "Fatigue Reliability Analysis of Contact Wire under Wind Load", Mechanical Science and Technology for Aerospace Engineering, vol. 30, no. 10, (2011), pp.1664-1668.

[11] W. Yu, "Fatigue Analysis of the External Gear Pump Axis Based on ANSYS", Fluid Power Transmission and Control, vol. 48, no. 5, (2011), pp.35-37.

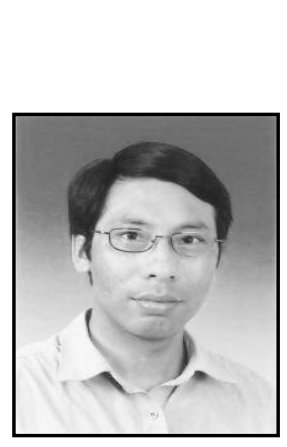

\section{Author}

Kuizhou Sun, man, associate professor, was born in Jinhu of Jiangsu Province in 1966. He research areas include reliability and optimization design. 